\title{
A APRENDIZAGEM BASEADA EM PROBLEMAS - PBL NO ENSINO REMOTO: APLICAÇÃO NA DISCPLINA DE PCIV.
}

DOI: 10.37702/2175-957X.COBENGE.2021.3619

SABRINA RODRIGUES GONCALVES - sabrinarg57@gmail.com

Universidade Federal do Ceará

Rafael Dantas 2236

60830-585 - Fortaleza - CE

BRUNO CAVALCANTE MOTA - bruno86925@gmail.com

UNIVERSIDADE FEDERAL DO CEARÁ

Rua Padre Alzir Sampaio 371

60355-260 - Fortaleza - CE

FRANCISCO HEBER LACERDA DE OLIVEIRA - heber@det.ufc.br UNIVERSIDADE FEDERAL DO CEARÁ

Centro de Tecnologia Bloco 703

60440-900 - Fortaleza - CE

Evanilson de Lima Siqueira - evanilsondelimasiqueira@gmail.com

Universidade Federal do Ceará

Rua Dom Xisto Albano 3854

60730-225 - Fortaleza - CE

JAIR CARVALHO FREIRE - jaircarvalhofreire123@gmail.com

UNIVERSIDADE FEDERAL DO CEARÁ

Avenida XXVI Res. Pacatuba II B 29 Ap 104

61800-000 - Pacatuba - CE

Resumo: Mesmo com os avanços tecnológicos, a rápida expansão da COVID-19 e sua chegada ao Brasil surpreendeu professores e estudantes ao tornar obrigatória e urgente a migração para um modelo de aulas remotas. Como consequência do isolamento social, as aulas presenciais foram suspensas nas instituições de ensino superior no Brasil, e o modelo de ensino remoto emergencial precisou ser posto em prática para dar continuidade às atividades pedagógicas. No contexto do ensino de engenharia, integrar a teoria à prática, estimular a solução de problemas reais e aproximar os estudantes dos desafios que eles encontrarão no mercado 
como forma de motivá-los já eram tópicos debatidos e com a adoção do ensino remoto essas questões ficam ainda mais expostas. Portanto, o objetivo deste artigo é apresentar a experiência da disciplina de Projeto e Construção da Infraestrutura Viária - PCIV, com abordagem de Aprendizagem Baseada em Projetos (PBL), ofertada de maneira totalmente remota pelo Departamento de Engenharia de Transportes da UFC no período de 2020.2, bem como o resultado da pesquisa realizada com os discentes que cursaram PCIV neste semestre com o intuito de captar a percepção destes acerca da metodologia da disciplina. Dessa forma, reforça-se a importância da adoção de diferentes metodologias de ensino-aprendizagem que busquem romper com os modelos de ensino tradicional e principalmente, que despertem nos discentes à aquisição de competências e habilidades que serão requeridas no mercado de trabalho. Tais aspectos se mostram ainda mais relevantes no modelo de ensino remoto, onde a motivação dos estudantes é ainda mais desafiada.

Palavras-chave: Ensino Remoto. PBL. Engenharia. 


\section{A APRENDIZAGEM BASEADA EM PROJETOS NO ENSINO REMOTO: APLICAÇÃO NA DISCPLINA DE PROJETO E CONSTRUÇÃO DA INFRAESTRUTURA VIÁRIA}

\section{INTRODUÇÃO}

As crescentes evoluções tecnológicas têm revolucionado a maneira como a sociedade se comunica e a velocidade com que se adquire informações, com impactos diretos no contexto do ensino e da aprendizagem. No entanto, mesmo com os avanços da tecnologia, a rápida expansão da COVID-19 e sua chegada ao Brasil surpreendeu professores e estudantes ao tornar obrigatória e urgente a migração para um modelo de aulas remotas.

Como consequência do isolamento social que visava atenuar a rápida transmissão do vírus, as aulas presenciais foram suspensas nas instituições de ensino superior no Brasil em março de 2020, e o modelo de ensino remoto emergencial (ERE) precisou ser posto em prática como uma resposta temporária para viabilizar as atividades pedagógicas (ALVES et al., 2020).

Assim, os professores migraram para plataformas como Youtube, Google Meet, Zoom, Microsoft Teams, Google Classroom dentre outras, buscando dar continuidade ao ensino que havia sido interrompido. Essas ferramentas viabilizam a realização das aulas, permitem que as mesmas fiquem gravadas para que o aluno assista posteriormente, além do compartilhamento de materiais didáticos que forneçam suporte à construção do aprendizado. No entanto, restringir o uso dessas plataformas a aulas meramente expositivas não garante a construção do conhecimento e a motivação do estudante. Para Moreira et al. (2020), essas tecnologias estão sendo utilizadas em uma perspectiva meramente instrumental, reduzindo metodologias e as práticas a um ensino apenas transmissivo, sendo necessário evoluir para uma educação digital em rede de qualidade.

Nesse sentido, Mota et al. (2020) cita como algumas desvantagens do ensino remoto, no tocante aos cursos de engenharia na Universidade Federal do Ceará, a utilização inadequada dos recursos tecnológicos, a ausência de infraestrutura para a realização e acompanhamento das aulas e a desmotivação por parte de alunos que não se adaptem ao modelo. Para Souza e Miranda (2020) um dos grandes desafios enfrentados pelo ensino remoto diz respeito à efetividade da aprendizagem, pois estar conectado não significa, necessariamente, dedicação às aulas online.

Integrar a teoria à prática, estimular a solução de problemas reais e aproximar os estudantes dos desafios que eles encontrarão no mercado como forma de motivá-los já eram tópicos discutidos no âmbito do ensino de engenharias. Com a adoção do ensino remoto essas questões ficaram ainda mais expostas. Somam-se a isso as Diretrizes Nacionais Curriculares (DCNs) dos cursos de graduação em engenharia (BRASIL, 2019) que demandam dos alunos, ao longo de sua formação a capacidade de conceber e projetar soluções criativas, além de realizar a avaliação crítico-reflexiva dos impactos das soluções de engenharia nos contextos social, econômico e ambiental.

Nesse contexto, surgem diferentes abordagens de ensino-aprendizagem com 0 intuito de suprir essas necessidades, dentre elas a Aprendizagem Baseada em Projetos (ABP) ou, do inglês, Project-Based Learning (PBL). Para Oliveira et al. (2020) o uso de técnicas como a aprendizagem baseada em projetos, foram introduzidas nas salas de aula para melhorar as habilidades de resolução de problemas dos alunos. Com isso, o objetivo deste artigo é avaliar a percepção dos discentes perante a experiência da disciplina de 
Projeto e Construção da Infraestrutura Viária (PCIV), que foi ofertada de maneira totalmente remota em 2020.2, com a abordagem da Aprendizagem Baseada em Projetos (ABP).

\section{ESTUDO DE CASO: A DISCPLINA DE PCIV}

\subsection{A aprendizagem baseada em projetos na disciplina de PCIV}

A disciplina de Projeto e Construção da Infraestrutura Viária (PCIV) é ofertada aos alunos do $6^{\circ}$ semestre do curso de Engenharia Civil da Universidade Federal do Ceará (UFC). Por meio dela, os alunos tem contato com projeto geométrico de rodovias e de terraplenagem. Dentre os objetivos, espera-se que os discentes sejam capazes de: (i) planejar e calcular os elementos geométricos de estradas; (ii) conhecer as etapas de construção da terraplenagem de estradas e (iii) desenvolver as etapas de um projeto de infraestrutura de vias de transporte.

Uma das metodologias aplicadas no desenvolvimento da disciplina é a Aprendizagem Baseada em Projeto (ABP). Segundo Cipolla (2016), a aprendizagem baseada em projetos, permite que diferentes grupos de estudantes confrontem questões e problemas do mundo real significativos para eles, determinando assim a maneira mais prática de abordá-los e estabelecer uma ação cooperativa em busca de soluções. Para Ghislandi (2017 apud Fernandes, 2014), a abordagem visa a melhora da aprendizagem discente e os prepara para a prática profissional. Dessa forma, em paralelo com as aulas teóricas acerca dos conceitos e cálculos envolvidos no projeto de infraestrutura de rodovias, os alunos são divididos em equipes de no máximo de 3 alunos para realizar o projeto de uma estrada, sendo realizado em duas etapas, na qual a primeira corresponde ao projeto geométrico e a segunda ao projeto de terraplenagem.

Cada equipe recebe uma quadrícula de um terreno (Figura 1) com alguns pontos que representam empreendimento ou áreas de proteção ambiental. $O$ objetivo dos estudantes é unir dois polos geradores de viagem presentes na quadrícula por meio de um traçado que seja econômico e sustentável, utilizando para este fim o software Civil 3D.

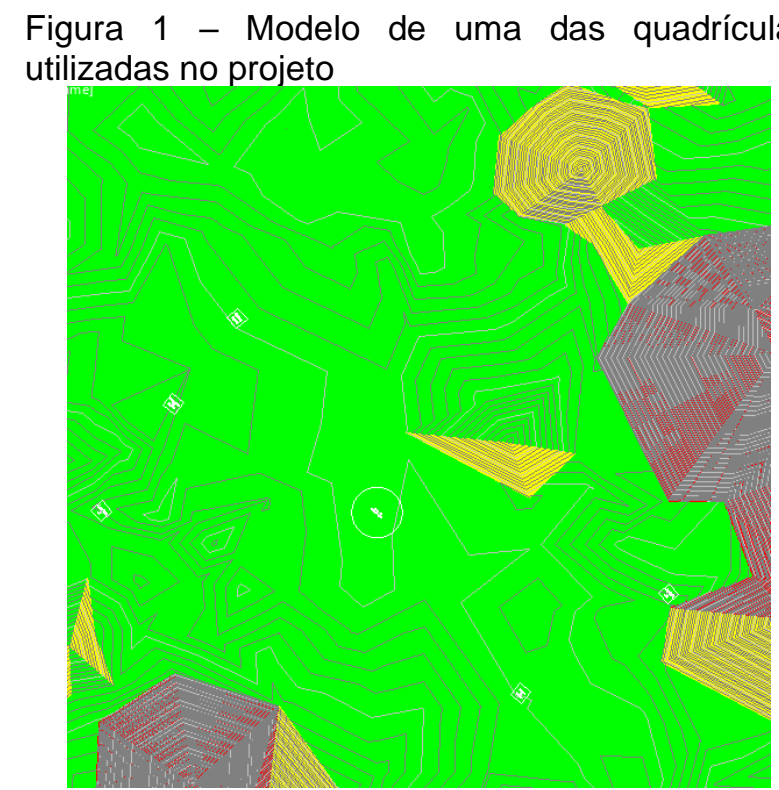

Fonte: Os autores (2021).

Para o auxílio ao uso da ferramenta de modelagem Civil 3D, são utilizadas 11 vídeo aulas disponibilizadas no canal Logística e Redes de Infraestrutura. Cada vídeo contém os 
passos a serem realizados no software. As aulas foram gravadas por monitores anteriores da disciplina e a versão utilizada é o Civil 3D 2018. Os temas dos vídeos são listados no Quadro 1. Durante o desenvolvimento do projeto, os alunos têm liberdade para tomarem, juntos a suas equipes, decisões projetuais relativas ao traçado, a escolha dos elementos das curvas horizontais e verticais, do greide do projeto, bem como das linhas de compensação no projeto de terraplenagem, embasando-se por normas e manuais do Departamento Nacional de Infraestrutura de Transportes (DNIT), aproximando-os do desenvolvimento de um projeto real.

Quadro 1 - Conteúdos das videoaulas práticas para desenvolvimento do projeto

\begin{tabular}{|c|c|}
\hline Aula & Título das videoaulas \\
\hline 01 & Mapa de Declividade - AutoCad Civil 3D \\
\hline 02 & Criação e configuração de um alinhamento - AutoCad Civil 3D \\
\hline 03 & Curvas Horizontais Simples e com Transição - AutoCad Civil 3D \\
\hline 04 & Superelevação - AutoCad Civil 3D \\
\hline 05 & Superlargura - AutoCad Civil 3D \\
\hline 06 & Perfil Longitudinal e Curvas Verticais - AutoCad Civil 3D \\
\hline 07 & Plotagem - AutoCad Civil 3D \\
\hline 08 & Seção Transversal - AutoCad Civil 3D \\
\hline 09 & Corredor - AutoCad Civil 3D \\
\hline 10 & Diagrama de Bruckner - AutoCad Civil 3D \\
\hline 11 & Diagrama de Bruckner e Linha de Compensação - AutoCad Civil 3D \\
\hline
\end{tabular}

Fonte: Os autores (2021).

\subsection{A abordagem remota da disciplina de PCIV no semestre 2020.2}

Inicialmente foi criado um canal na plataforma Youtube para serem disponibilizadas as aulas, de forma que, os encontros ocorriam duas vezes por semana no horário corrente da disciplina pela plataforma Google Meet, sendo gravados. Após a gravação, os vídeos eram editados e disponibilizados no canal Monitoria PCIV 2020.2 no Youtube. Ao todo, foram postados vídeos dos 24 encontros da disciplina (ver Figura 2). As aulas estão disponíveis em 03 playlists que contemplam os tópicos gerais da disciplina: (i) projeto geométrico; (ii) projeto de terraplenagem e (iii) custos de serviço de terraplenagem.

Figura 2 - Canal no Youtube para disponibilização das aulas

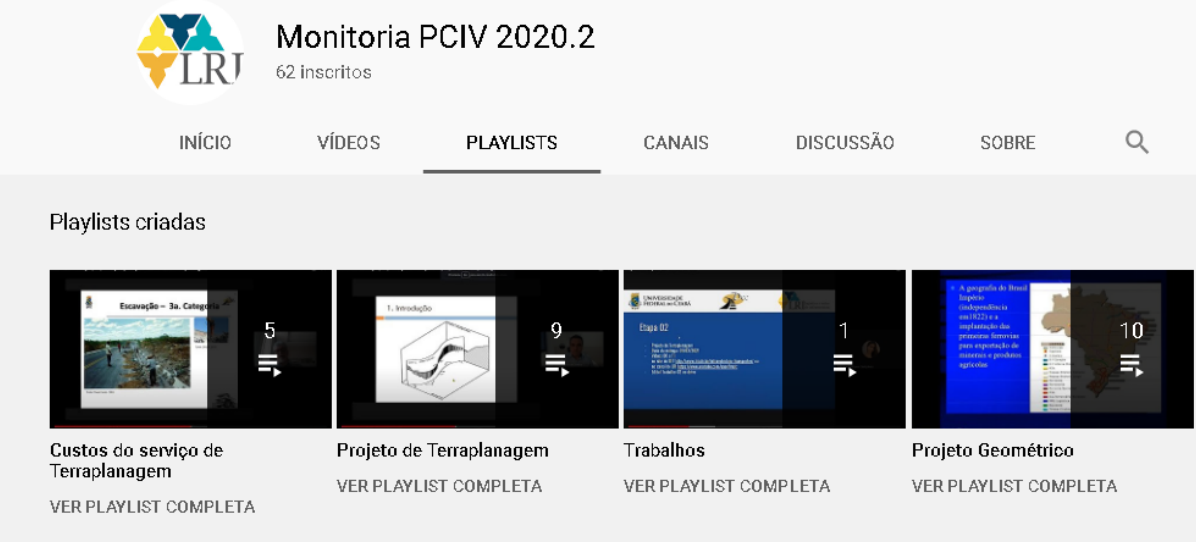

Fonte: Os autores (2021). 


\subsection{A abordagem remota da monitoria de PCIV}

A monitoria exerce impacto no ensino superior, sendo definida por Ortolan et al. (2019) como uma atividade em que discentes de graduação - os monitores - participam ativamente no ensino de colegas de turmas posteriores às suas, desenvolvendo atividades como elaboração de questões, atendimento a dúvidas e discussão sobre elementos próprios da disciplina e do fazer pedagógico. A disciplina de Projeto e Construção da Infraestrutura Viária tem o suporte de bolsistas do Programa de Iniciação à Docência (PID), que auxiliam os estudantes com o desenvolvimento do projeto. Os impactos da suspensão das aulas e encontros presenciais também foram sentidos diretamente pelos alunos monitores, pois estes, assim como os professores, também precisaram se reinventar e achar meios de continuarem às atividades de suporte aos alunos que cursavam a disciplina.

Antes de ser instaurado o ensino remoto emergencial, os encontros da monitoria da disciplina de PCIV ocorriam de forma presencial e tinham o objetivo de discutir sobre a realização do trabalho, tirar dúvidas sobre a ferramenta Civil 3D e resolver questões. Com a suspensão das aulas, essas reuniões passaram a ocorrer de forma remota por meio da plataforma Google Meet e os alunos agendavam horários com os monitores através da ferramenta Doodle (ver Figura 3).

Figura 3 - Interface da ferramenta Doodle

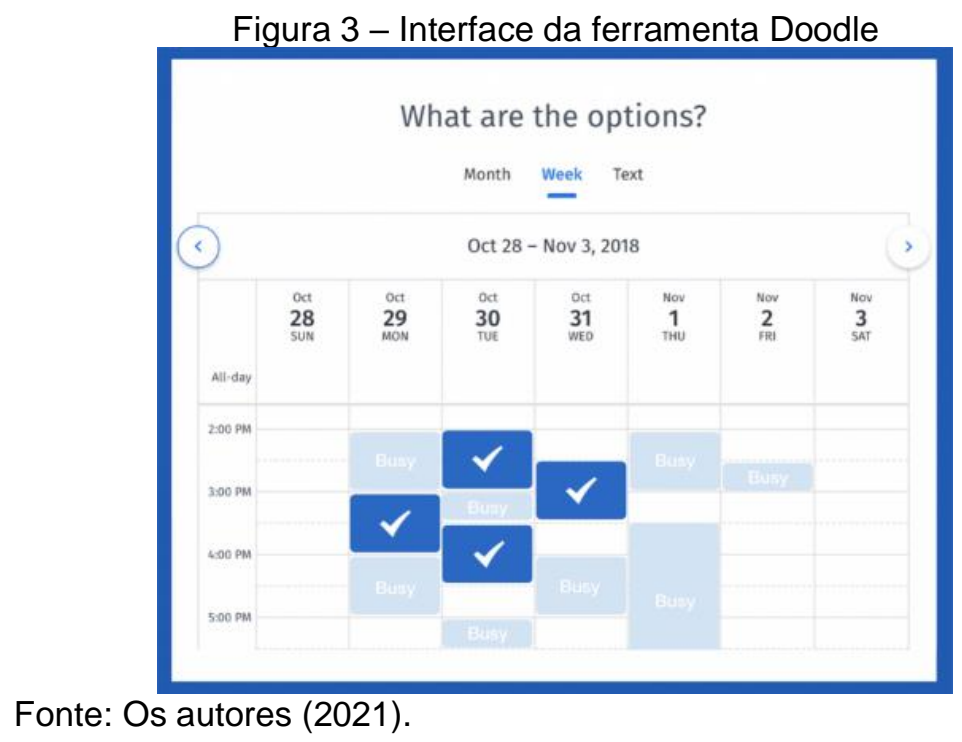

\section{MÉTODO DE PESQUISA}

A metodologia deste trabalho consistiu em um estudo de caso do desenvolvimento da disciplina de PCIV no ano de 2020.2 de forma totalmente remota e com o suporte da Aprendizagem Baseada em Projetos (ABP) e na aplicação de um questionário ao final do semestre letivo com o intuito de levantar a percepção dos estudantes acerca da estratégia aplicada. O questionário foi respondido por 47 estudantes, onde os participantes deveriam avaliar em uma escala de 1 a 5 , que variava de "discordo totalmente" a "concordo totalmente", afirmações relativas às atividades desenvolvidas no decorrer da disciplina.

\section{RESULTADOS E DISCUSSÕES}

A pesquisa realizada ao final da disciplina reafirmou a importância da monitoria no suporte da disciplina. $90 \%$ dos alunos acreditam totalmente que ela foi importante no suporte ao desenvolvimento do trabalho. Acerca da plataforma utilizada pelos monitores 
(Figura 4), apesar de a maioria dos discentes estarem totalmente contemplados pelo Google Meet para os encontros de monitoria, a porcentagem de $18 \%$ dos discentes que não estão totalmente satisfeitos pode ser explicada por algumas dificuldades em auxiliar na solução de problemas no Civil 3D sem mexer diretamente no computador do aluno. Para sanar este problema, pode-se analisar a viabilidade de serem utilizadas ferramentas de controle remoto.

Figura 4 - Nível de satisfação dos discentes com a plataforma para monitorias

\section{Estou satisfeito com plataforma utilizada para as monitorias (Google Meet)}

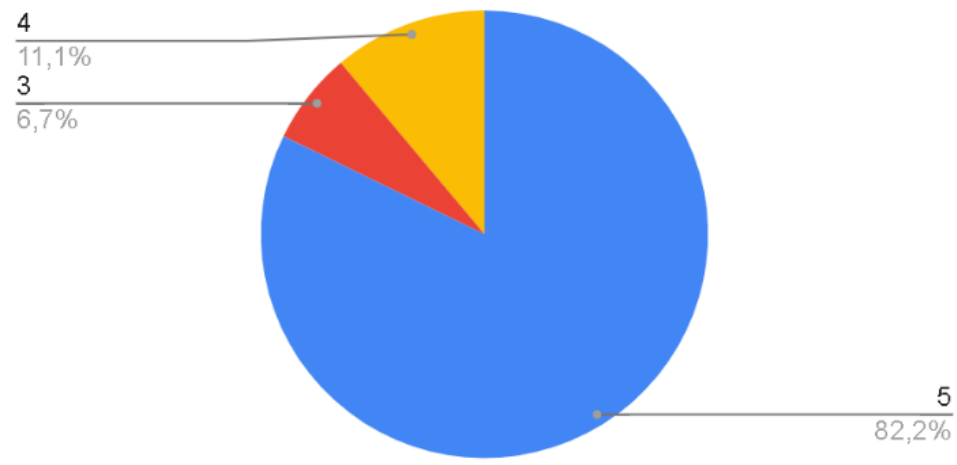

Fonte: Os autores (2021).

No tocante às videoaulas do Civil 3D, a maioria dos estudantes concorda totalmente que estas foram importantes no desenvolvimento do projeto (Figura 5). Contudo, recomenda-se que as vídeo aulas sejam atualizadas para a versão mais atual do software.

Figura 5 - Percepção dos discentes sobre a importância das vídeo aulas no desenvolvimento do projeto

As vídeo aulas do Civil-3D foram importantes no suporte ao desenvolvimento do projeto

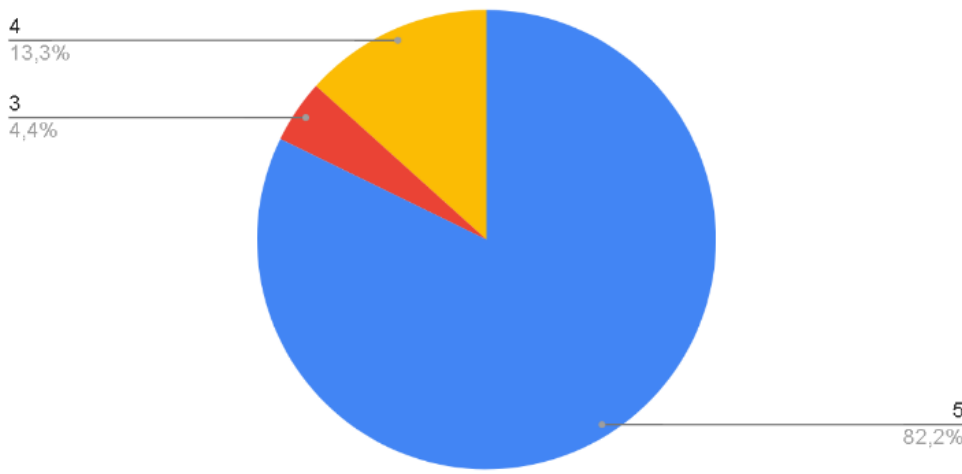

Fonte: Os autores (2021).

Por fim, $70 \%$ dos alunos concordam totalmente que o desenvolvimento do projeto contribuiu para consolidar os assuntos vistos nas aulas (Figura 6). Apenas $2 \%$ dos alunos discordam parcialmente dessa afirmativa. Esse resultado corrobora com um dos pilares da ABP de que o aprendizado pode ser construído pela prática.

Figura 6 - Percepção dos discentes sobre o projeto prático 
desenvolvimento de um projeto durante a disciplina ajudou a consolidar os assuntos vistos nas aulas

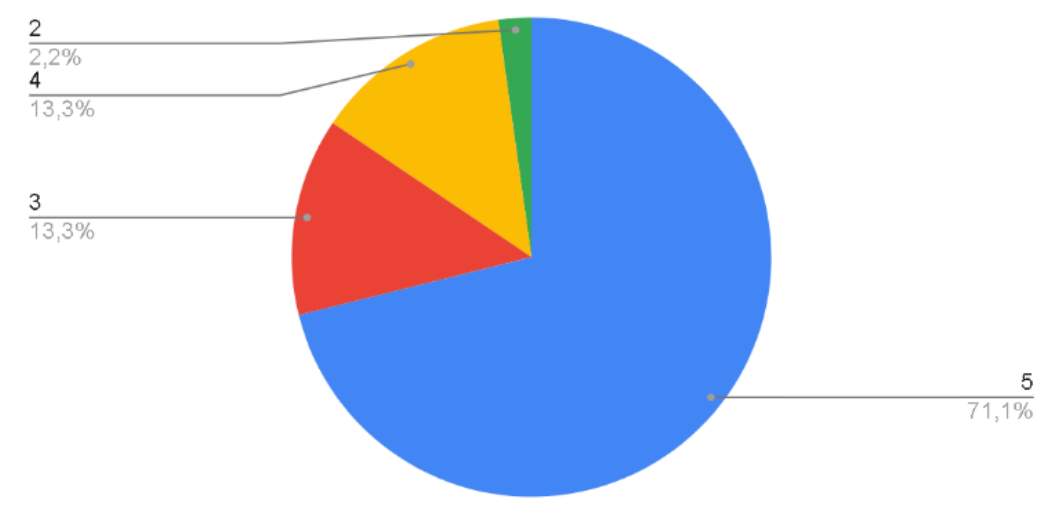

Fonte: Os autores (2021).

\section{CONSIDERAÇÕES FINAIS}

O presente trabalho reforça a importância da adoção de metodologias distintas de ensino-aprendizagem que busquem romper com os modelos de ensino tradicional e principalmente, que despertem nos discentes à aquisição de competências e habilidades que serão requeridas no mercado de trabalho e os aproximem da vivência prática. Tais questões se mostram ainda mais relevantes no modelo de ensino remoto, no qual a motivação dos estudantes é ainda mais desafiada.

Neste artigo, a Aprendizagem Baseada em Projetos (ABP) é mostrada como uma dessas metodologias, colocando os alunos para, em grupos, executarem um projeto de infraestrutura de uma rodovia, oferecendo-os contato com ferramentas utilizadas atualmente e com um ambiente de estímulo a soluções de engenharia. $\mathrm{O}$ artigo apresenta também como o modelo de ensino remoto pode ser positivo para a comunidade acadêmica como um todo, uma vez que estão sendo disponibilizados cada vez mais vídeos e materiais nas plataformas online abertas ao público como o Youtube, permitindo maior troca de informações e experiências.

Além disso, reforça-se a importância das atividades de monitoria, principalmente no modelo remoto, como agente facilitadora da aprendizagem e sugere-se o uso de diferentes ferramentas de controle remoto pelos monitores de disciplinas práticas, como o anydesk ou o teamviewer para facilitar a interação com o projeto do aluno. Por fim, a disciplina de PCIV revela que a existência de uma prática aliada aos conhecimentos teóricos pode contribuir para consolidar os conhecimentos vistos nas aulas.

\section{Agradecimentos}

Os autores agradecem à UFC pela concessão de bolsa de monitoria pelo Programa de Iniciação à Docência (PID), ao CNPq pela bolsa de mestrado do penúltimo autor, ao grupo de Logística e Redes de Infraestruturas (LRI) por todo o suporte no desenvolvimento da disciplina e aos alunos, que contribuíram participando da pesquisa.

\section{REFERÊNCIAS}

ALMEIDA, Mara Cristina; DA SILVA, Silvia Sonia; BARRIOS, Scarlet Karina Montilla. Aprendizagem Baseada em Projetos no Curso de Engenharia Civil: na Visão de 
Diferentes Instituições, Docentes e Acadêmicos. Revista Pleiade, v. 14, n. 30, p. 71-78, 2020.

\section{ALVES, João Marcelo et al. ENSINO A DISTÂNCIA: CARACTERÍSTICAS E DESAFIOS.} In: Congresso Nacional Universidade, EAD e Software Livre. Anais. 2020.

BRASIL. Ministério da Educação. RESOLUÇÃO № 2, DE 24 DE ABRIL DE 2019 https://www.in.gov.br/en/web/dou/-/resolu\%C3\%87\%C3\%83o-n\%C2\%BA-2-de-24-deabril-de-2019-85344528. Acesso: 22/04/2021

CIPOLLA, L. E. Aprendizagem baseada em projetos: a educação diferenciada para o século XXI. Tradução: Fernando de Siqueira Rodrigues, Porto Alegre: Penso, 2015. Escrito por William N. Bender. Administração: Ensino E Pesquisa, 17(3), 567-585, 2016. https://doi.org/10.13058/raep.2016.v17n3.440

GHISLANDI, Marcos G. et al. Aprendizagem Baseada em Projetos: Um Estudo das Experiências Implementadas no Campus das Engenharias da Universidade Federal Rural de Pernambuco. GRAPHIC DESIGN, p. 30, 2017.

MOREIRA, J. António; HENRIQUES, Susana; BARROS, Daniela Melaré Vieira. Transitando de um ensino remoto emergencial para uma educação digital em rede, em tempos de pandemia. Dialogia, p. 351-364, 2020.

MOTA, Bruno $\mathrm{C}$, et al. Estratégias de ensino remoto e a comparação com o Ensino presencial na área de engenharia de transportes. In: XLVIII Congresso Brasileiro de Educação em Engenharia. Anais. 2020.

OLIVEIRA, João Lucas et al. Sala de aula 4.0-Uma proposta de ensino remoto baseado em sala de aula invertida, gamification e PBL. Revista Brasileira de Informática na Educação, v. 28, p. 909-933, 2020.

ORTOLAN, Lucas; ALTEFF, Luciana França; TIBURZIO, Vera Lúcia Bonfim. A importância e os desafios da monitoria universitária na formação docente: um relato de experiência. Revista de Ensino de Biologia da SBEnBio, p. 289-308, 2020.

SOUZA, Dominique Guimarães; MIRANDA, Jean Carlos. Desafios da implementação do ensino remoto. Boletim de Conjuntura (BOCA), v. 4, n. 11, p. 81-89, 2020.

\section{PROJECT-BASED LEARNING IN REMOTE LEARNING: APPLICATION IN PCIV SUBJECT}

Abstract: Even with the technological advances, the rapid expansion of COVID-19 and its arrival in Brazil surprised professors and students by making the migration to a remote classroom model mandatory and urgent. As a consequence of social isolation, face-to-face classes were suspended at higher education institutions in Brazil, and the emergency remote teaching model needed to be put in place to provide continuity to pedagogical activities. In the context of engineering education, integrating theory and practice, stimulating the solution of real problems, and bringing students closer to the challenges they will face in the market as a way to motivate them were already debated topics, and with the 
adoption of remote teaching these issues became even more exposed. Therefore, the objective of this article is to evaluate the students' perception of the experience of the discipline Design and Construction of Road Infrastructure, which was offered entirely remotely, with a Project-Based Learning (PBL) approach. The research method was based on the presentation of the case study and the application of a questionnaire to the students of the course. This reinforces the importance of adopting different teaching-learning methodologies that seek to break with traditional teaching models and, above all, that encourage students to acquire the competencies and skills that will be required in the job market. Such aspects are even more relevant in the remote teaching model, in which the motivation of students is even more challenged

Keywords: Remote Learning. PBL. Civil Engineering. 